\title{
STOCHASTIC MODELING FOR ENVIRONMENTAL STRESS SCREENING
}

\author{
JI HWAN CHA, ${ }^{*}$ Ewha Womans University \\ MAXIM FINKELSTEIN, ${ }^{* *}$ University of the Free State and University ITMO
}

\begin{abstract}
Environmental stress screening (ESS) of manufactured items is used to reduce the occurrence of future failures that are caused by latent defects by eliminating the items with these defects. Some practical descriptions of the relevant ESS procedures can be found in the literature; however, the appropriate stochastic modeling and the corresponding thorough analysis have not been reported. In this paper we develop a stochastic model for the ESS, analyze the effect of this operation on the population characteristics of the screened items, and also consider the relevant optimization issues.
\end{abstract}

Keywords: Environmental stress screening; burn-in; stress-strength model; shock model; nonhomogeneous Poisson process

2010 Mathematics Subject Classification: Primary 60K10

Secondary 62P30

\section{Introduction}

There are different ways of improving reliability characteristics of manufactured items. The most common methodology adopted in industry is burn-in, which is a method of 'elimination' of initial failures (infant mortality). Usually, to burn-in a component or a system means to subject it to a fixed time period of simulated use prior to actual operation. Due to the high failure rate during the early stages of the component's life, burn-in has been widely accepted as an effective method of screening out these initial failures. Thus, the 'sufficient condition' for employing the traditional burn-in is the initially decreasing failure rate. For example, when a population of items is heterogeneous and, therefore, consists of subpopulations with ordered failure (hazard) rates, it obviously contains weaker (with larger failure rates) subpopulations. As the weakest populations die out first (see Finkelstein (2008)), the failure rate of this population is often initially decreasing and burn-in can be effectively applied (see Mi (1994a), (1994b), (1996) and Cha and Finkelstein (2010)).

It should be noted that not all populations of engineering items that contain 'weaker' items to be eliminated exhibit this shape of failure rate. For example, the 'weakness' of some manufactured items can result from the latent defects that can create additional failure modes. The failure rate in this case is not necessarily decreasing (see Example 1) and, therefore, traditional burn-in should not be applied. However, by applying the short-time excessive stress, the weaker items in the population with increasing failure rate can be eliminated by the environmental stress screening (ESS) and, therefore, the reliability characteristics of the

Received 5 October 2012; revision received 20 June 2013.

* Postal address: Department of Statistics, Ewha Womans University, Seoul, 120-750, Korea.

Email address: jhcha@ewha.ac.kr

** Postal address: Department of Mathematical Statistics, University of the Free State, 339 Bloemfontein 9300, South

Africa. Email address: finkelm@ufs.ac.za 
population of items that have successfully passed the ESS test can still improve. This is the crucial distinction between this operation and burn-in. In fact, the formal difference between the ESS and burn-in has not been clearly defined in the literature. In this paper we understand the ESS as the method of elimination of items with additional (nonconventional) failure modes, whereas burn-in targets elimination of weaker items with conventional failure modes and it is effective only when the population failure rate is initially decreasing. Another important distinction of the proposed model from burn-in is that the ESS can also create new defects in items that were previously defect free.

Numerous stochastic models of burn-in have been intensively studied in the literature during the last decades (see Mi (1991) and Cha (2009) for the corresponding review). Although some practical engineering approaches to the ESS modeling were reported (see, e.g. Fiorentino and Saari (1983) and Yan and English (1997)), to the best of the authors' knowledge, there has been little research dealing with adequately advanced stochastic modeling and analysis of the ESS.

In this paper we develop a stochastic model for the ESS, analyze its effect on the population characteristics of the screened items, and describe related optimization problems. We assume that, due to substandard materials or a faulty manufacturing process, some of the manufactured items are susceptible to additional causes of failure (failure mode), i.e. shocks (such as electrical or mechanical shocks). We define the ESS as a procedure of applying a shock of controlled magnitude, i.e. a short-time excessive stress. In practice, for example, a shock can be understood as a short-time electric impulse. For the ESS to be effective, the corresponding magnitude should be reasonably larger than the magnitude of shocks that occur in field usage.

Our modeling is within the framework of the general stress-strength model. Indeed, the failure of an item occurs when the magnitude of the stress (shock) exceeds its strength. A larger ESS shock magnitude (within 'physical limits') implies better reliability characteristics of survived items in field usage, but at the same time, incurs a larger ESS cost as more items with defects are discarded. An important feature of our model is that we assume that the item during field usage is exposed to a point process of environmental shocks with an ordinary, not excessive magnitude. These shocks can obviously destroy only defective items that have passed the ESS or were induced by the ESS.

The paper is organized as follows. In Section 2 we provide a general supplementary result for the stress-strength model that will be used intensively throughout this paper. Section 3 is devoted to the detailed probabilistic analysis of the suggested model. In Section 4 we discuss and illustrate the corresponding optimization problem. Finally, some concluding remarks are given in the last section.

\section{General stress-strength-type shock model}

In this section we consider a rather general stress-strength shock model, which will be used as a crucial supplementary result for considering the ESS model of the subsequent sections. For other related shock models, see, e.g. Cha and Finkelstein (2011).

Consider a system (item) subject to the nonhomogeneous Poisson process (NHPP) of shocks $N(t), t \geq 0$, with rate $\lambda(t)$ and arrival times $T_{i}, i=1,2, \ldots$ Let, for simplicity, shocks be the only cause of a system's failure. We assume that the initial shock process, $N(t)$, $t \geq 0$, is external for the system in the sense that $N(t), t \geq 0$, may influence the system's performance but is not influenced by it (see Fleming and Harrington (1991)). Denote by $S_{i}$ the magnitude (stress) of the $i$ th shock. Assume that $S_{i}, i=1,2, \ldots$, are independent and identically distributed (i.i.d.) random variables with common cumulative distribution function $(\mathrm{CDF}) M(s)=\operatorname{Pr}\left(S_{i} \leq s\right)(\bar{M}(s) \equiv 1-M(s))$ and corresponding probability density function 
(PDF) $m(s)$. Let $U$ be a random strength of the system with the corresponding CDF, survival function (SF), PDF, and failure rate (FR) respectively denoted by $G_{U}(u), \bar{G}_{U}(u), g_{U}(u)$, and $r_{U}(u)$. For each $i=1,2, \ldots$, the operable system survives if $S_{i} \leq U$ and fails if $S_{i}>U$, independently of everything else. Thus, we are interested in the probability of survival of our system in $[0, t), t>0$.

Let $T_{S}$ be the lifetime of the system described above, and let $\lambda_{S}(t)$ be the corresponding FR function. Note that a more general discussion of the history-dependent shock models can be found in Cha and Finkelstein (2012), (2013). However, for the sake of presentation and further applications to ESS problems, the following result will be obtained in the described specific setting only.

Theorem 1. The failure rate function of the described system corresponding to $T_{S}$ is given by

$$
\lambda_{S}(t)=p(t) \lambda(t)
$$

where

$$
p(t) \equiv \mathbb{P}\left(S_{N(t-)+1}>U \mid T_{S} \geq t\right)=\frac{\int_{0}^{\infty} \int_{0}^{v} \exp \left\{-\bar{M}(r) \int_{0}^{t} \lambda(x) \mathrm{d} x\right\} g_{U}(r) \mathrm{d} r m(v) \mathrm{d} v}{\int_{0}^{\infty} \exp \left\{-\bar{M}(r) \int_{0}^{t} \lambda(x) \mathrm{d} x\right\} g_{U}(r) \mathrm{d} r} .
$$

Proof. Assume first that $N(t), t \geq 0$, is a general, orderly shock process. Let $H_{t-} \equiv\{N(s)$, $0 \leq s<t\}$ be its history up to $t$. Then the conditional probability of failure in the infinitesimal interval of time can be formally written as

$$
\begin{aligned}
\mathbb{P}\left(T_{S} \in\right. & {\left.[t, t+\mathrm{d} t) \mid T_{S} \geq t, H_{t-}, U\right) } \\
= & \mathbb{P}\left(T_{S} \in[t, t+\mathrm{d} t) \mid T_{S} \geq t, H_{t-}, U, N((t+\mathrm{d} t)-)-N(t-)=1\right) \\
& \times \mathbb{P}\left(N((t+\mathrm{d} t)-)-N(t-)=1 \mid T_{S} \geq t, H_{t-}, U\right) \\
& +\mathbb{P}\left(T_{S} \in[t, t+\mathrm{d} t) \mid T_{S} \geq t, H_{t-}, U, N((t+\mathrm{d} t)-)-N(t-)=0\right) \\
& \quad \times \mathbb{P}\left(N((t+\mathrm{d} t)-)-N(t-)=0 \mid T_{S} \geq t, H_{t-}, U\right) \\
= & \mathbb{P}\left(T_{S} \in[t, t+\mathrm{d} t) \mid T_{S} \geq t, H_{t-}, U, N((t+\mathrm{d} t)-)-N(t-)=1\right) \\
& \times \mathbb{P}\left(N((t+\mathrm{d} t)-)-N(t-)=1 \mid H_{t-}\right),
\end{aligned}
$$

where

$$
\mathbb{P}\left(N((t+\mathrm{d} t)-)-N(t-)=1 \mid T_{S} \geq t, H_{t-}, U\right)
$$

reduces to

$$
\mathbb{P}\left(N((t+\mathrm{d} t)-)-N(t-)=1 \mid H_{t-}\right)
$$

as $H_{t-}$ completely defines the corresponding conditional probability due to our assumption that the initial shock process is external. Accordingly, from (1),

$$
\begin{aligned}
\mathbb{P}\left(T_{S}\right. & \left.\in[t, t+\mathrm{d} t) \mid T_{S} \geq t, H_{t-}, U\right) \\
& =\mathbb{P}\left(T_{S} \in[t, t+\mathrm{d} t) \mid T_{S} \geq t, H_{t-}, U, N((t+\mathrm{d} t)-)-N(t-)=1\right) v\left(t \mid H_{t-}\right) \mathrm{d} t,
\end{aligned}
$$

where $v\left(t \mid H_{t-}\right)$ is the conditional intensity function of a point process (see Cox and Isham (1980) and Andersen et al. (1993)) defined by

$$
v\left(t \mid H_{t-}\right) \equiv \lim _{\Delta t \rightarrow 0} \frac{\mathbb{P}\left(N((t+\Delta t)-)-N(t-)=1 \mid H_{t-}\right)}{\Delta t} .
$$


For the NHPP, obviously, $v\left(t \mid H_{t-}\right)=\lambda(t)$. It is also clear that

$$
\mathbb{P}\left(T_{S} \in[t, t+\mathrm{d} t) \mid T_{S} \geq t, H_{t-}, U, N((t+\mathrm{d} t)-)-N(t-)=1\right)=\mathbf{1}\left(S_{N(t-)+1}>U\right),
$$

where $\mathbf{1}(\cdot)$ is the corresponding indicator. Therefore, (2) now becomes

$$
\mathbb{P}\left(T_{S} \in[t, t+\mathrm{d} t) \mid T_{S} \geq t, H_{t-}, U\right)=\mathbf{1}\left(S_{N(t-)+1}>U\right) \lambda(t) \mathrm{d} t,
$$

and, thus

$$
\mathbb{P}\left(T_{S} \in[t, t+\mathrm{d} t) \mid T_{S} \geq t\right)=\mathbb{E}\left[\mathbf{1}\left(S_{N(t-)+1}>U\right)\right] \lambda(t) \mathrm{d} t,
$$

where the expectation is with respect to the conditional distribution of $U \mid T_{S} \geq t$. From (3), $\lambda_{S}(t)$ is given by

$$
\lambda_{S}(t)=\mathbb{P}\left(S_{N(t-)+1}>U \mid T_{S} \geq t\right) \lambda(t) .
$$

As the distribution of $S_{N(t-)+1}$ does not depend on the event $\left\{T_{S} \geq t\right\}$, it is sufficient to derive the distribution for $U \mid T_{S} \geq t$. Observe that

$$
\mathbb{P}\left(T_{S} \geq t \mid N(t-)=n, U=u\right)=\mathbb{P}\left(u \geq \max \left\{S_{1}, S_{2}, \ldots, S_{n}\right\}\right)=(M(u))^{n} .
$$

Thus,

$$
\begin{aligned}
\mathbb{P}\left(T_{S} \geq t, U>u\right) & =\int_{u}^{\infty} \sum_{n=0}^{\infty}(M(r))^{n} \frac{\left(\int_{0}^{t} \lambda(x) \mathrm{d} x\right)^{n}}{n !} \exp \left\{-\int_{0}^{t} \lambda(x) \mathrm{d} x\right\} g_{U}(r) \mathrm{d} r \\
& =\int_{u}^{\infty} \exp \left\{-\bar{M}(r) \int_{0}^{t} \lambda(x) \mathrm{d} x\right\} g_{U}(r) \mathrm{d} r
\end{aligned}
$$

and

$$
\mathbb{P}\left(U>u \mid T_{S} \geq t\right)=\frac{\int_{u}^{\infty} \exp \left\{-\bar{M}(r) \int_{0}^{t} \lambda(x) \mathrm{d} x\right\} g_{U}(r) \mathrm{d} r}{\int_{0}^{\infty} \exp \left\{-\bar{M}(r) \int_{0}^{t} \lambda(x) \mathrm{d} x\right\} g_{U}(r) \mathrm{d} r} .
$$

Therefore, from (4) we have

$$
\lambda_{S}(t)=\frac{\int_{0}^{\infty} \int_{0}^{v} \exp \left\{-\bar{M}(r) \int_{0}^{t} \lambda(x) \mathrm{d} x\right\} g_{U}(r) \mathrm{d} r m(v) \mathrm{d} v}{\int_{0}^{\infty} \exp \left\{-\bar{M}(r) \int_{0}^{t} \lambda(x) \mathrm{d} x\right\} g_{U}(r) \mathrm{d} r} \lambda(t) .
$$

As stated, our system survives if $S_{i} \leq U$ and fails if $S_{i}>U$ for each $i=1,2, \ldots$, independently of everything else. Thus, at first glance, one may expect that the corresponding failure probability $p(t)$ is given by $p(t) \equiv \mathbb{P}\left(S_{N(t-)+1}>U\right)$ (cf. Brown and Proschan (1983)). However, from Theorem 1, $p(t)$ is given by $\mathbb{P}\left(S_{N(t-)+1}>U \mid T_{S} \geq t\right)$. Observe that the proof of Theorem 1 is based on a procedure similar to a Bayesian approach when the strength of an item is updated according to information on the survival up to a current time. The understanding of this 'Bayesian reasoning' behind our model will be crucial for our further discussions in the next sections.

\section{Stochastic model for ESS}

The description and assumptions of our model are as follows. During the manufacturing process, items with failure rate $r(t)$ and corresponding lifetime $T_{N}$ (which is due only to the 'normal' failure mode), and also defective items with lifetime $T_{D}$ are produced. Let the proportion of the nondefective items be $\pi$ and that of the defective items be $1-\pi$. 
The defective items, in addition to the normal failure mode of the nondefective items, are characterized by a new additional failure mode. In this paper we assume that this additional failure mode describes susceptibility to external shocks. For example, consider the case when the normal (nondefective) items, in accordance with specifications, should not be susceptible to electrical or mechanical shocks. However, due to substandard materials or a faulty manufacturing process, some of the produced items are susceptible to these shocks (see Yan and English (1997)). For instance, during the manufacturing process, the items can be exposed to a strong electric shock and this shock may result in some defective items which are even sensitive to electrical shocks of a 'normal' magnitude, whereas the nondefective items are not sensitive to them (see Landers et al. (1994)). Another example is when a small crack in the material of a defective item is sensitive to mechanical impulses (e.g. vibration) in field use, which can eventually result in its failure. Thus, we assume that shocks of a 'normal' magnitude also occur in field operations and, therefore, the defective items can fail due to this failure mode. On the other hand, the nondefective items do not fail from external shocks of this type in field operations as they do not have the corresponding failure mode.

In accordance with our description, the survival function of $T_{N}$ is

$$
\mathbb{P}\left(T_{N}>t\right)=\exp \left\{-\int_{0}^{t} r(u) \mathrm{d} u\right\} .
$$

Let the two failure modes of the defective items be independent. Then, the corresponding survival function is given by the competing risks model (series system):

$$
\mathbb{P}\left(T_{D}>t\right)=\exp \left\{-\int_{0}^{t} r(u) \mathrm{d} u\right\} \mathbb{P}\left(T_{E}>t\right) .
$$

Here $T_{E}$ is the lifetime that accounts only for the external shock failure mode.

Suppose that during the field operation the external shocks occur in accordance with the NHPP $N(t), t \geq 0$, with rate $\lambda(t)$. As in Section 2, denote by $S_{i}$ the magnitude (stress) of the $i$ th shock and assume that $S_{i}, i=1,2, \ldots$, are i.i.d. random variables with the common CDF $M(s)=\operatorname{Pr}\left(S_{i} \leq s\right)(\bar{M}(s) \equiv 1-M(s))$ and corresponding PDF $m(s)$. The defective item is characterized by its random strength $U$, i.e. the resistance ability to external shocks. Here the strength is understood as the maximum stress level that the defective item can survive. The corresponding CDF, SF, PDF, and FR of $U$ are respectively denoted by $G_{U}(u), \bar{G}_{U}(u), g_{U}(u)$, and $r_{U}(u)$. For each $i=1,2, \ldots$, the operable system survives if $S_{i} \leq U$ and fails if $S_{i}>U$, independently of everything else. Then, in accordance with Theorem 1, (5) now reads

$$
\mathbb{P}\left(T_{D}>t\right)=\exp \left\{-\int_{0}^{t} r(u) \mathrm{d} u\right\} \exp \left\{-\int_{0}^{t} p(u) \lambda(u) \mathrm{d} u\right\},
$$

where

$$
p(t) \equiv \frac{\int_{0}^{\infty} \int_{0}^{v} \exp \left\{-\bar{M}(r) \int_{0}^{t} \lambda(x) \mathrm{d} x\right\} g_{U}(r) \mathrm{d} r m(v) \mathrm{d} v}{\int_{0}^{\infty} \exp \left\{-\bar{M}(r) \int_{0}^{t} \lambda(x) \mathrm{d} x\right\} g_{U}(r) \mathrm{d} r} .
$$

From (6) we see that the lifetimes of the nondefective and defective items are obviously stochastically ordered: $T_{D}<_{\mathrm{fr}} T_{N}$, where ' $<_{\mathrm{fr}}$ ' denotes the failure (hazard) rate ordering of two random variables (see Shaked and Shanthikumar (2007)). 
Denote the population lifetime by $T$. As it consists of defective and nondefective items with given proportions, the corresponding survival function is the following mixture

$$
\begin{aligned}
\bar{F}(t) & \equiv \mathbb{P}(T>t) \\
& =\pi \exp \left\{-\int_{0}^{t} r(u) \mathrm{d} u\right\}+(1-\pi) \exp \left\{-\int_{0}^{t} r(u) \mathrm{d} u\right\} \exp \left\{-\int_{0}^{t} p(u) \lambda(u) \mathrm{d} u\right\} .
\end{aligned}
$$

Thus, (8) defines the survival function of an item in field usage that is chosen at random from the population of manufactured items.

In what follows, we will describe the impact of the ESS on the population structure and on the corresponding population lifetime distribution. Therefore, we must first define the ESS that we consider in this paper.

Definition 1. (ESS process.) During the ESS, all items are exposed to a single shock with fixed magnitude $s$. If the strength of a defective item is larger than $s$ then it survives; otherwise, it fails. Depending on the magnitude $s$, a proportion of nondefective items, $\rho(s), 0 \leq \rho(s)<1$, becomes defective, where $\rho(s)$ is an increasing function of its argument. The items failed during the ESS are discarded and only the survived items are put into the field operation.

Thus, the ESS, in principle, can induce defects. Furthermore, as those with induced defects but not failed are not identifiable, they are also put into the field operation.

Recall that the shock magnitudes in field operations are i.i.d. random variables. We assume that the corresponding mean is substantially smaller than the magnitude of stress allowed for the ESS (otherwise, there is no reason to perform the ESS). Therefore, the shocks in field operations can hardly 'produce' defective items out of nondefective items (or this effect is negligible). On the other hand, these shocks can still destroy the defective item with a given strength.

Denote by $T_{\text {ESS }}$ the population lifetime after the ESS with magnitude $s$.

Theorem 2. Under the above assumptions, the population distribution and the corresponding failure rate (after the ESS) are given by

$$
\bar{F}_{E}(t, s)=\mathbb{P}\left(T_{\mathrm{ESS}}>t\right)=\sum_{i=1}^{3} \pi(i) \bar{F}_{i}(t)
$$

and

$$
\begin{aligned}
\lambda_{E}(t, s)= & r(t) \frac{\pi(1) \bar{F}_{1}(t)}{\sum_{i=1}^{3} \pi(i) \bar{F}_{i}(t)}+[r(t)+p(t) \lambda(t)] \frac{\pi(2) \bar{F}_{2}(t)}{\sum_{i=1}^{3} \pi(i) \bar{F}_{i}(t)} \\
& +[r(t)+p(s, t) \lambda(t)] \frac{\pi(3) \bar{F}_{3}(t)}{\sum_{i=1}^{3} \pi(i) \bar{F}_{i}(t)},
\end{aligned}
$$

where

$$
\begin{aligned}
& \bar{F}_{1}(t) \equiv \exp \left\{-\int_{0}^{t} r(u) \mathrm{d} u\right\} \\
& \pi(1) \equiv \frac{(1-\rho(s)) \pi}{(1-\rho(s)) \pi+\rho(s) \pi+\bar{G}_{U}(s)(1-\pi)} \\
& \bar{F}_{2}(t) \equiv \exp \left\{-\int_{0}^{t} r(u) \mathrm{d} u\right\} \exp \left\{-\int_{0}^{t} p(u) \lambda(u) \mathrm{d} u\right\}
\end{aligned}
$$




$$
\begin{aligned}
\pi(2) & \equiv \frac{\rho(s) \pi}{(1-\rho(s)) \pi+\rho(s) \pi+\bar{G}_{U}(s)(1-\pi)}, \\
\bar{F}_{3}(t) & \equiv \exp \left\{-\int_{0}^{t} r(u) \mathrm{d} u\right\} \exp \left\{-\int_{0}^{t} p(s, u) \lambda(u) \mathrm{d} u\right\} \\
\pi(3) & \equiv \frac{\bar{G}_{U}(s)(1-\pi)}{(1-\rho(s)) \pi+\rho(s) \pi+\bar{G}_{U}(s)(1-\pi)},
\end{aligned}
$$

and

$$
p(s, t)=\frac{\int_{s}^{\infty} \int_{s}^{v} \exp \left\{-\bar{M}(r) \int_{0}^{t} \lambda(x) \mathrm{d} x\right\} g_{U}(r) \mathrm{d} r m(v) \mathrm{d} v}{\int_{s}^{\infty} \exp \left\{-\bar{M}(r) \int_{0}^{t} \lambda(x) \mathrm{d} x\right\} g_{U}(r) \mathrm{d} r} .
$$

Proof. Observe that there are now three subpopulations after the ESS and we can define the corresponding frailty variable $Z$ :

- the subpopulation with nondefective items $(Z=1)$;

- the subpopulation with defective items which were originally nondefective $(Z=2)$;

- the subpopulation with defective items which were originally defective but have survived the $\operatorname{ESS}(Z=3)$.

Then, in accordance with our notation, the distribution of $Z$ is given by

$$
\begin{aligned}
& \pi(1) \equiv \mathbb{P}(Z=1)=\frac{(1-\rho(s)) \pi}{(1-\rho(s)) \pi+\rho(s) \pi+\bar{G}_{U}(s)(1-\pi)}, \\
& \pi(2) \equiv \mathbb{P}(Z=2)=\frac{\rho(s) \pi}{(1-\rho(s)) \pi+\rho(s) \pi+\bar{G}_{U}(s)(1-\pi)}, \\
& \pi(3) \equiv \mathbb{P}(Z=3)=\frac{\bar{G}_{U}(s)(1-\pi)}{(1-\rho(s)) \pi+\rho(s) \pi+\bar{G}_{U}(s)(1-\pi)} .
\end{aligned}
$$

Therefore,

$$
\bar{F}_{1}(t) \equiv \mathbb{P}\left(T_{\mathrm{ESS}}>t \mid Z=1\right)=\exp \left\{-\int_{0}^{t} r(u) \mathrm{d} u\right\}
$$

and

$$
\bar{F}_{2}(t) \equiv \mathbb{P}\left(T_{\mathrm{ESS}}>t \mid Z=2\right)=\exp \left\{-\int_{0}^{t} r(u) \mathrm{d} u\right\} \exp \left\{-\int_{0}^{t} p(u) \lambda(u) \mathrm{d} u\right\},
$$

where $p(t)$ is given by $(7)$.

The derivation of $\mathbb{P}\left(T_{\mathrm{ESS}}>t \mid Z=3\right)$ is not so straightforward. Indeed, it should be taken into account that, when we apply a shock of controlled magnitude $s$ during the ESS, this means that the strength of the defective item that had passed the ESS is larger than $s$ and, therefore, the distribution of the remaining strength $U_{s}$ (given that the strength is larger than $s$ ) is

$$
G_{U}(u \mid s) \equiv \mathbb{P}(U \leq u \mid U>s)=1-\frac{\bar{G}(u)}{\bar{G}(s)}, \quad u>s .
$$


Thus, the function $p(t)$ in (7) should be modified to

$$
\begin{aligned}
p(s, t) & =\frac{\int_{0}^{\infty} \int_{0}^{v} \exp \left\{-\bar{M}(r) \int_{0}^{t} \lambda(x) \mathrm{d} x\right\} g_{U}(r \mid s) \mathrm{d} r m(v) \mathrm{d} v}{\int_{0}^{\infty} \exp \left\{-\bar{M}(r) \int_{0}^{t} \lambda(x) \mathrm{d} x\right\} g_{U}(r \mid s) \mathrm{d} r} \\
& =\frac{\int_{s}^{\infty} \int_{s}^{v} \exp \left\{-\bar{M}(r) \int_{0}^{t} \lambda(x) \mathrm{d} x\right\} g_{U}(r) \mathrm{d} r m(v) \mathrm{d} v}{\int_{s}^{\infty} \exp \left\{-\bar{M}(r) \int_{0}^{t} \lambda(x) \mathrm{d} x\right\} g_{U}(r) \mathrm{d} r},
\end{aligned}
$$

where $g_{U}(u \mid s)$ is the corresponding PDF of $G_{U}(u \mid s)$, which is given by

$$
g_{U}(u \mid s)= \begin{cases}0 & \text { if } u \leq s \\ \frac{g_{U}(u)}{\bar{G}_{U}(s)} & \text { if } u>s\end{cases}
$$

Finally,

$$
\bar{F}_{3}(t) \equiv \mathbb{P}\left(T_{\text {ESS }}>t \mid Z=3\right)=\exp \left\{-\int_{0}^{t} r(u) \mathrm{d} u\right\} \exp \left\{-\int_{0}^{t} p(s, u) \lambda(u) \mathrm{d} u\right\} .
$$

Therefore, (9) and (10) hold.

We will now discuss the effect of the ESS on the quality of the population after the screening by comparing $\bar{F}_{E}(t, s)$ to the survival function without screening, $F(t)$ defined by (8). As the ESS in our model can create defective items, theoretically, in some cases this operation may have a negative effect on the population of items.

Definition 2. The severity (stress) level $s$ is said to be inadmissible under the survival function criterion if $\bar{F}(t) \geq \bar{F}_{E}(t, s)$ for all $t>0$. Otherwise, the severity (stress) level $s$ is said to be admissible.

Obviously, the inadmissible severity levels should not be considered in the ESS practice as the reliability of items in field use is worse than that without the ESS in this case. Note that the condition for the 'admissibility' in Definition 2 means that $\bar{F}(t)<\bar{F}_{E}(t, s)$ for some $t>0$ and not for all $t>0$. However, for obvious practical reasons, we are mostly interested in the latter case. The following definition addresses this setting.

Definition 3. The severity (stress) level $s$ is said to be positively admissible under the survival function criterion if $\bar{F}(t)<\bar{F}_{E}(t, s)$ for all $t>0$.

Theorem 3. (i) If $\rho(s)<(1-\pi) G_{U}(s)$ then the severity level s is positively admissible under the survival function criterion.

(ii) If $\rho(s) \pi \geq \pi(1-\pi)+(1-\pi)^{2} \bar{G}_{U}(s)$ then the severity level $s$ is inadmissible under the survival function criterion.

Proof. Define, for convenience, $\lambda_{1}(t) \equiv r(t), \lambda_{2}(t) \equiv r(t)+p(t) \lambda(t)$, and $\lambda_{3}(t) \equiv r(t)+$ $p(s, t) \lambda(t)$. Note that (11) can be written in a compact and a meaningful way as

$$
p(s, t)=\int_{0}^{\infty} \mathbf{1}(v \in[s, \infty)) h(s, t, v) m(v) \mathrm{d} v,
$$

where

$$
h(s, t, v) \equiv \frac{\int_{s}^{v} \exp \left\{-\bar{M}(r) \int_{0}^{t} \lambda(x) \mathrm{d} x\right\} g_{U}(r) \mathrm{d} r}{\int_{s}^{\infty} \exp \left\{-\bar{M}(r) \int_{0}^{t} \lambda(x) \mathrm{d} x\right\} g_{U}(r) \mathrm{d} r}
$$


and $\mathbf{1}(\cdot)$ is the corresponding indicator. Observe that, for all fixed $t$ and $v$,

$$
\begin{aligned}
\frac{\partial}{\partial s} h(s, t, v) & \\
= & \frac{1}{\left(\int_{s}^{\infty} \exp \left\{-\bar{M}(r) \int_{0}^{t} \lambda(x) \mathrm{d} x\right\} g_{U}(r) \mathrm{d} r\right)^{2}} \\
& \times\left[-\exp \left\{-\bar{M}(s) \int_{0}^{t} \lambda(x) \mathrm{d} x\right\} g_{U}(s) \int_{s}^{\infty} \exp \left\{-\bar{M}(r) \int_{0}^{t} \lambda(x) \mathrm{d} x\right\} g_{U}(r) \mathrm{d} r\right. \\
& \left.\quad+\exp \left\{-\bar{M}(s) \int_{0}^{t} \lambda(x) \mathrm{d} x\right\} g_{U}(s) \int_{s}^{v} \exp \left\{-\bar{M}(r) \int_{0}^{t} \lambda(x) \mathrm{d} x\right\} g_{U}(r) \mathrm{d} r\right] \\
< & 0
\end{aligned}
$$

for all $s>0$. Therefore, the function $p(s, t)$ is strictly decreasing in $s$ for each fixed $t$. This implies that $p(s, t)<p(t)$ for all $t>0$ and $s>0$. Thus, we have the failure rate ordering

$$
\lambda_{1}(t)<\lambda_{3}(t)<\lambda_{2}(t) \text { for all } t>0,
$$

and, accordingly,

$$
\bar{F}_{1}(t)>\bar{F}_{3}(t)>\bar{F}_{2}(t) \text { for all } t>0,
$$

where $\bar{F}_{i}(t) \equiv \exp \left\{-\int_{0}^{t} \lambda_{i}(u) \mathrm{d} u\right\}, i=1,2,3$. Observe that, in accordance with (8),

$$
\bar{F}(t)=\pi \bar{F}_{1}(t)+(1-\pi) \bar{F}_{2}(t),
$$

whereas, in accordance with (9),

$$
\bar{F}_{E}(t, s)=\pi(1) \bar{F}_{1}(t)+\pi(2) \bar{F}_{2}(t)+\pi(3) \bar{F}_{3}(t) .
$$

Therefore, if $\pi(2)+\pi(3)<1-\pi$, or, equivalently, $\pi(1)>\pi$, then

$$
\begin{aligned}
\bar{F}_{E}(t, s)-\bar{F}(t) & >(\pi(1)-\pi) \bar{F}_{1}(t)+\left[\pi(2) \bar{F}_{2}(t)+\pi(3) \bar{F}_{2}(t)-(1-\pi) \bar{F}_{2}(t)\right] \\
& =(\pi(1)-\pi) \bar{F}_{1}(t)-(\pi(1)-\pi) \bar{F}_{2}(t) \\
& >0
\end{aligned}
$$

for all $t>0$. The condition $\pi(2)+\pi(3)<1-\pi$ is equivalent to $\rho(s)<(1-\pi) G_{U}(s)$. This completes the proof of (i).

By a similar reasoning, if $\pi(2) \geq 1-\pi$, or, equivalently, $\rho(s) \pi \geq \pi(1-\pi)+(1-\pi)^{2} \bar{G}_{U}(s)$, then the severity level $s$ is inadmissible under the survival function criterion.

Remark 1. (i) The conditions of Theorem 3 do not imply the admissibility/inadmissibility of the corresponding severity level under the failure rate criterion. That is, the condition $\pi(2)+\pi(3)<1-\pi$ does not imply that $\lambda_{T}(t)>\lambda_{E}(t, s)$ for all $t>0$, where $\lambda_{T}(t)$ is the failure rate which corresponds to $\bar{F}(t)$ defined in (8).

(ii) The failure rate ordering (12) will be important for further reasoning later. This ordering implies that the quality of defective items improves after the ESS, but they are still obviously 'worse' than the nondefective items. 
Remark 2. The effect of applying two consecutive shocks with severity $s$ during the ESS can also be considered. After this type of ESS, we also have three subpopulations with failure rates $\lambda_{1}(t)=r(t), \lambda_{2}(t)=r(t)+p(t) \lambda(t)$, and $\lambda_{3}(t)=r(t)+p(s, t) \lambda(t)$, and corresponding proportions

$$
\begin{aligned}
& \pi^{(2)}(1)=\frac{(1-\rho(s)) \pi(1)}{(1-\rho(s)) \pi(1)+\rho(s) \pi(1)+\left[\pi(3)+\bar{G}_{U}(s) \pi(2)\right]}, \\
& \pi^{(2)}(2)=\frac{\rho(s) \pi(1)}{(1-\rho(s)) \pi(1)+\rho(s) \pi(1)+\left[\pi(3)+\bar{G}_{U}(s) \pi(2)\right]}, \\
& \pi^{(2)}(3)=\frac{\pi(3)+\bar{G}_{U}(s) \pi(2)}{(1-\rho(s)) \pi(1)+\rho(s) \pi(1)+\left[\pi(3)+\bar{G}_{U}(s) \pi(2)\right]} .
\end{aligned}
$$

\section{Optimal severity}

In this section we consider the problem of determining the optimal severity level (magnitude) of the ESS. Let $\tau$ be the mission time of an item in the field operation. If it does not fail during this time then the mission is considered to be successful. Thus, the probability of the mission success needs to be maximized and we should find the optimal severity level $s^{*}$ that satisfies

$$
\bar{F}_{E}\left(\tau, s^{*}\right)=\max _{s>0} \bar{F}_{E}(\tau, s) .
$$

Alternatively, let MRL $(s)$ denote the mean time to failure of an item in the field operation as a function of $s$, i.e. $\operatorname{MRL}(s) \equiv \int_{0}^{\infty} \bar{F}_{E}(t, s) \mathrm{d} t$. Then, the optimal severity level which maximizes the mean time to failure should be obtained as

$$
\operatorname{MRL}\left(s^{*}\right)=\max _{s>0} \operatorname{MRL}(s) .
$$

For defining the optimal severity, we consider the admissible severity class rather than the positively admissible class as we have to take into account all admissible severity levels. It is often more convenient to describe the dual inadmissible class. The following theorem provides the upper bound for the optimal severity level that maximizes the mission success probability or mean time to failure in field usage.

Theorem 4. Suppose that $\rho(\infty) \equiv \lim _{s \rightarrow \infty} \rho(s)>1-\pi$, and let

$$
s_{0} \equiv \inf _{s \geq 0}\left\{s: \rho(s) \pi \geq \pi(1-\pi)+(1-\pi)^{2} \bar{G}_{U}(s)\right\} .
$$

Then the severity levels in $\left(s_{0}, \infty\right)$ are inadmissible. Therefore, $s_{0}$ is the upper bound for the optimal severity level.

Proof. From Theorem 3, the condition for inadmissibility is

$$
\rho(s) \pi \geq \pi(1-\pi)+(1-\pi)^{2} \bar{G}_{U}(s) .
$$

Here the function $\rho(s) \pi$ is increasing from 0 to $\rho(\infty) \pi$, whereas the function $\pi(1-\pi)+(1-$ $\pi)^{2} \bar{G}_{U}(s)$ decreases from $(1-\pi)$ to $\pi(1-\pi)$. Thus, if $\rho(\infty) \pi>\pi(1-\pi)$, or, equivalently, $\rho(\infty)>(1-\pi)$, then there exists $s_{0} \in(0, \infty)$ such that the severity levels in $\left(s_{0}, \infty\right)$ are inadmissible. Therefore, $s_{0}$ is the upper bound for the optimal severity.

Remark 3. It is reasonable to assume that in practice $\lim _{s \rightarrow \infty} \rho(s)=1$ and that the proportion of the defective items, $1-\pi$, is relatively small. Therefore, the condition $\rho(\infty)>1-\pi$ can be satisfied in almost all practical cases. 


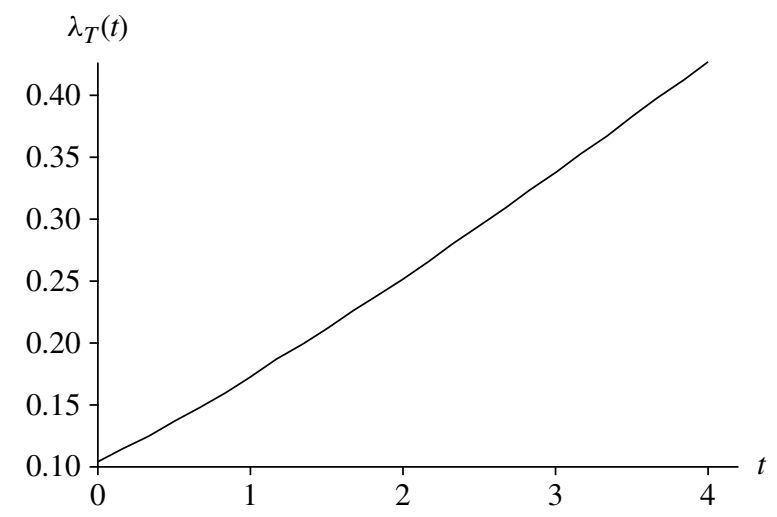

Figure 1: The graph of $\lambda_{T}(t)$.

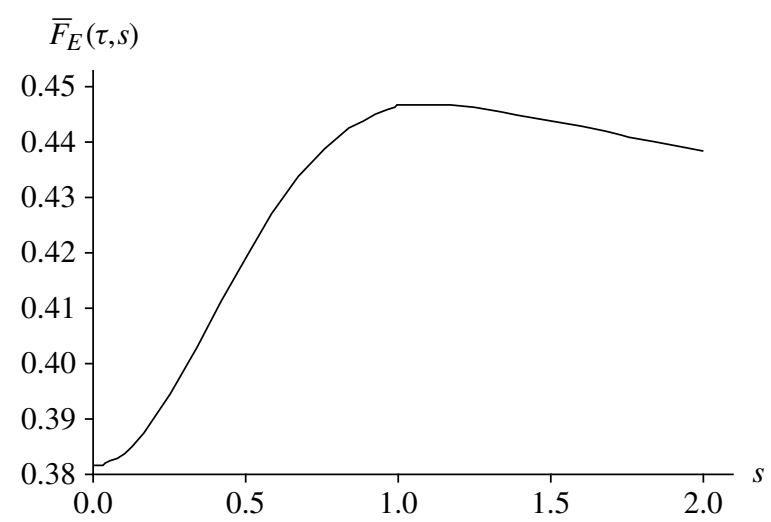

Figure 2: The graph of $\bar{F}_{E}(\tau, s)$.

Example 1. Let $r(t)=0.1 t, t \geq 0, \lambda(t)=1, t \geq 0, m(s)=3 \exp \{-3 s\}, s \geq 0, g_{U}(u)=$ $4 u \exp \left\{-2 u^{2}\right\}, u \geq 0, \pi=0.7, \tau=4.0$, and

$$
\rho(s)= \begin{cases}0, & 0 \leq s<1, \\ 1-\exp \{-0.05(s-1)\}, & s \geq 1 .\end{cases}
$$

In Figure 1 we plot the failure rate of the population distribution before the ESS, which is obtained based on (8). Therefore, as $\lambda_{T}(t)$ is increasing, the burn-in procedure should not be applied to this population. On the other hand, as $\rho(s)$ is strictly increasing for $s \geq 1$, there exists a unique solution to the equation

$$
\rho(s) \pi=\pi(1-\pi)+(1-\pi)^{2} \bar{G}_{U}(s),
$$

which is the upper bound for the optimal severity level. Therefore, the ESS as a method of elimination of defective items is justified in this case. Solving this equation numerically results in $s_{0} \approx 8.13$. Therefore, it is now sufficient to search for the optimal severity level in the interval $[0,8.13]$. The graph of $\bar{F}_{E}(\tau, s)$ is given in Figure 2. The optimal severity level in this case is $s^{*} \approx 1.08$ and the maximum probability of the mission success is $\bar{F}_{E}\left(\tau ; s^{*}\right) \approx 0.447$. 
Based on the above results, we now consider certain cost structures for determining the cost-based optimal severity level. As previously, an item is chosen at random from our initial population and is exposed to a shock of magnitude $s$ during the ESS. If it survives, it is put into the field operation; otherwise, the failed item is discarded and a new item is chosen from the population, etc. This procedure is repeated until the first survived item is obtained. Let $c_{s r}$ be the shop replacement cost (actually, it is the cost of a new item), and let $c_{s}$ be the cost for conducting the ESS. Let $c_{1}(s)$, as a function of $s$, be the expected cost for eventually obtaining a component which has survived the ESS. Then

$$
c_{1}(s)=\frac{c_{s}+c_{s r}\left[1-\left\{\pi+(1-\pi) \bar{G}_{U}(s)\right\}\right]}{\pi+(1-\pi) \bar{G}_{U}(s)},
$$

where $1 /\left[\pi+(1-\pi) \bar{G}_{U}(s)\right]$ is the total number of trials until the first 'success'.

Assume that if a mission (of length $\tau$ ) is successful (in the field operation) then the gain $K$ is 'earned'; otherwise, a penalty $C$ is imposed, where $K>C>0$. Then the expected cost during the field operation is

$$
c_{2}(s)=-K \bar{F}_{E}(\tau, s)+C F_{E}(\tau, s)=-(K+C) \bar{F}_{E}(\tau, s)+C
$$

and the total expected cost $c(s)$ is

$$
\begin{aligned}
c(s) & =c_{1}(s)+c_{2}(s) \\
& =\frac{c_{s}+c_{s r}\left[1-\left\{\pi+(1-\pi) \bar{G}_{U}(s)\right\}\right]}{\pi+(1-\pi) \bar{G}_{U}(s)}-(K+C) \bar{F}_{E}(\tau ; s)+C .
\end{aligned}
$$

The objective is now to find the optimal severity level $s^{*}$ that satisfies

$$
s^{*}=\arg \min _{s \in[0, \infty]} c(s) .
$$

Similar to Theorem 4 , if $\rho(\infty) \equiv \lim _{s \rightarrow \infty} \rho(s)>1-\pi$, then the optimal severity level which minimizes $c_{2}(s)$ (maximizes $\bar{F}_{E}(\tau, s)$, as follows from (13)) does not exist in the interval $\left(s_{0}, \infty\right)$, where $s_{0}$ is also defined by Theorem 4 . Furthermore, as $c_{1}(s)$ is strictly increasing to $\infty$, we can conclude that the optimal severity level $s^{*}$ should exist in the interval $\left[0, s_{0}\right]$.

Assume now that during field operation the gain is proportional to the mean time to failure. Therefore, the total average cost function in this case is

$$
c(s)=\frac{c_{s}+c_{s r}\left[1-(1-\pi) \bar{G}_{U}(s)\right]}{(1-\pi) \bar{G}_{U}(s)}-K \int_{0}^{\infty} \bar{F}_{E}(t ; s) \mathrm{d} t .
$$

By similar arguments, the optimal severity level $s^{*}$ should exist in the interval $\left[0, s_{0}\right]$.

\section{Concluding remarks}

The ESS is an important tool for increasing reliability characteristics and for decreasing costs in usage for the manufactured items with latent defects. In contrast to burn-in, for which numerous models and applications have been elaborated and reported in the literature, the ESS has not been so formally described. One of the reasons for this is because burn-in usually mimics the actual usage of items in real time, whereas the ESS applies the excessive stress that can 'quickly' destroy the defected items and, therefore, does not resemble the actual usage 
at all. On the other hand, there are situations when the effect of the ESS can be described in similar terms as the effect of an environment on the item in field use. One of these situations is described in this paper using the stress-strength concept.

We assumed that the defective item possesses resistance to external stress, whereas the ESS applies stress that can destroy this item if its magnitude is large enough. We also assumed that in field use the items are subjected to the NHPP of shocks (although of the smaller magnitude) that can destroy the defective item as well. Therefore, we were able to discuss the joint model for ESS-field use in a unified way.

An important and novel feature of our modeling takes into account the fact that the ESS can also create new defects from items that were previously defect free. We assumed that the proportion of these items increases as the magnitude of the stress increases. Therefore, the corresponding optimization problem of obtaining an optimal magnitude of the ESS for maximizing characteristics in field use can be formulated and studied.

\section{Acknowledgements}

The authors would like to thank the anonymous referee for very kind and detailed suggestions on the presentation of this paper. The work of the first author was supported by a National Research Foundation of Korea (NRF) grant funded by the Korean government (MEST) (grant number 2011-0017338), and the Priority Research Centers Program through the National Research Foundation of Korea (NRF) funded by the Ministry of Education, Science and Technology (2009-0093827). The work of the second author was supported by the NRF (National Research Foundation of South Africa) under grant IFR2011040500026.

\section{References}

Andersen, P. K., Borgan, Ø., Gill, R. D. And Keiding, N. (1993). Statistical Models Based on Counting Processes. Springer, New York.

Brown, M. and Proschan, F. (1983). Imperfect repair. J. Appl. Prob. 20, 851-859.

Cha, J. H. (2009). Burn-in models: recent issues, development and future topics. Commun. Korean Statist. Soc. 16, $871-880$.

Cha, J. H. And Finkelstein, M. (2010). Burn-in by environmental shocks for two ordered subpopulations. Europ. J. Operat. Res. 206, 111-117.

Cha, J. H. And Finkelstein, M. (2011). On new classes of extreme shock models and some generalizations. J. Appl. Prob. 48, 258-270.

Cha, J. H. And Finkelstein, M. (2012). Information-based thinning of point processes and its application to shock models. J. Statist. Planning Infer. 142, 2345-2350.

Cha, J. H. And Finkelstein, M. (2013). On history-dependent shock models. Operat. Res. Lett. 41, $232-237$.

Cox, D. R. And Isham, V. (1980). Point Processes. Chapman \& Hall, London.

Finkelstein, M. (2008). Failure Rate Modelling for Reliability and Risk. Springer, London.

FIOREnTINo, E. AND SAARI, A. E. (1983). Planning of production reliability stress-screening programs. IEEE Trans. Reliab. 32, 247-252.

Fleming, T. R. and Harrington, D. P. (1991). Counting Processes and Survival Analysis. John Wiley, New York.

Landers, T. L., Malstrom, R. M., Schmitt, N. and Fant, E. (1994). Electronics Manufacturing Processes. Prentice Hall, Englewood Cliffs, NJ.

Mi, J. (1991). Optimal Burn-in. Doctoral Thesis, University of Pittsburgh.

Mi, J. (1994a). Burn-in and maintenance policies. Adv. Appl. Prob. 26, 207-221.

Mi, J. (1994b). Maximization of a survival probability and its application. J. Appl. Prob. 31, 1026-1033.

MI, J. (1996). Minimizing some cost functions related to both burn-in and field use. Operat. Res. 44, 497-500.

Shaked, M. and Shanthikumar, J. G. (2007). Stochastic Orders. Springer, New York.

YAN, L AND ENGLISH, J. R. (1997). Economic cost modeling of environmental-stress-screening and burn-in. IEEE Trans. Reliab. 46, 275-282. 